\title{
Coupled-resonator-based metamaterials
}

\author{
Masao Kitano ${ }^{\mathrm{a})}$, Yasuhiro Tamayama, \\ and Toshihiro Nakanishi \\ Department of Electronic Science and Engineering, Kyoto University \\ Kyoto-daigaku Katsura, Nishikyo-ku, Kyoto 615-8510, Japan \\ a)kitano@kuee.kyoto-u.ac.jp
}

\begin{abstract}
In this review, we describe recent developments in functional metamaterials based on coupled resonators. We first consider coupled resonator metamaterials that mimic electromagnetically induced transparency (EIT). We present a circuit model for EIT-like metamaterials and introduce a new coupled resonator in which the coupling is provided by a field gradient so that the group velocity can be varied by varying the incident angle. We then describe the principles for enhancing second harmonic generation (SHG) in nonlinear resonant metamaterials. Optical and microwave experiments of SHG in singly resonant metamaterials are presented. A method for further enhancing SHG using a doubly resonant metamaterial is also described.
\end{abstract}

Keywords: metamaterial, coupled resonator, electromagnetically induced transparency, group velocity, nonlinear optics, second harmonic generation

Classification: Electromagnetic theory

\section{References}

[1] R. E. Collin, Field Theory of Guided Waves, 2nd ed., IEEE Press, Piscataway, 1990.

[2] J. B. Pendry, A. J. Holden, D. J. Robbins, and W. J. Stewart, "Magnetism from Conductors and Enhanced Nonlinear Phenomena," IEEE Trans. Microw. Theory Tech., vol. 47, no. 11, pp. 2075-2084, Nov. 1999.

[3] R. A. Shelby, D. R. Smith, and S. Schultz, "Experimental Verification of a Negative Index of Refraction," Science, vol. 292, no. 5514, pp. 77-79, April 2001.

[4] J. B. Pendry, "Negative Refraction Makes a Perfect Lens," Phys. Rev. Lett., vol. 85, no. 18, pp. 3966-3969, Oct. 2000.

[5] Z. Jacob, L. V. Alekseyev, and E. Narimanov, "Optical Hyperlens: Farfield imaging beyond the diffraction limit," Opt. Express, vol. 14, no. 18, pp. 8247-8256, Sept. 2006.

[6] J. B. Pendry, D. Schurig, and D. R. Smith, "Controlling Electromagnetic Fields," Science, vol. 312, no. 5781, pp. 1780-1782, June 2006.

[7] M. Silveirinha and N. Engheta, "Tunneling of Electromagnetic Energy through Subwavelength Channels and Bends using $\varepsilon$-Near-Zero Materials," Phys. Rev. Lett., vol. 97, no. 15, 157403, Oct. 2006.

[8] Y. Tamayama, T. Nakanishi, K. Sugiyama, and M. Kitano, "An invisible medium for circularly polarized electromagnetic waves," Opt. Express, 
vol. 16, no. 25, pp. 20869-20875, Dec. 2008.

[9] Y. Tamayama, T. Nakanishi, K. Sugiyama, and M. Kitano, "NoReflection Phenomena for Chiral Media," Wave Propagation, ed. A. Petrin, Rijeka, InTech, pp. 415-432, 2011.

[10] S. E. Harris, "Electromagnetically Induced Transparency," Phys. Today, vol. 50, no. 7, pp. 36-42, July 1997.

[11] L. V. Hau, S. E. Harris, Z. Dutton, and C. H. Behroozi, "Light speed reduction to 17 metres per second in an ultracold atomic gas," Nature, vol. 397, no. 6720, pp. 594-598, Feb. 1999.

[12] M. Fleischhauer, A. Imamoglu, and J. P. Marangos, "Electromagnetically induced transparency: Optics in coherent media," Rev. Mod. Phys., vol. 77, no. 2, pp. 633-673, July 2005.

[13] B. E. A. Saleh and M. C. Teich, Fundamentals of Photonics, 2nd ed. Hoboken, Wiley-Interscience, 2007.

[14] V. A. Fedotov, M. Rose, S. L. Prosvirnin, N. Papasimakis, and N. I. Zheludev, "Sharp Trapped-Mode Resonance in Planar Metamaterials with a Broken Structural Symmetry," Phys. Rev. Lett., vol. 99, no. 14, 147401, Oct. 2007.

[15] S. Zhang, D. A. Genov, Y. Wang, M. Liu, and X. Zhang, "PlasmonInduced Transparency in Metamaterials," Phys. Rev. Lett., vol. 101, no. 4, 047401, July 2008.

[16] N. Papasimakis, V. A. Fedotov, N. I. Zheludev, and S. L. Prosvirnin, "Metamaterial Analog of Electromagnetically Induced Transparency," Phys. Rev. Lett., vol. 101, no. 25, 253903, Dec. 2008.

[17] N. Papasimakis, Y. H. Fu, V. A. Fedotov, S. L. Prosvirnin, D. P. Tsai, and N. I. Zheludev, "Metamaterial with polarization and direction insensitive resonant transmission response mimicking electromagnetically induced transparency," Appl. Phys. Lett., vol. 94, no. 21, 211902, May 2009.

[18] P. Tassin, L. Zhang, T. Koschny, E. N. Economou, and C. M. Soukoulis, "Low-Loss Metamaterials Based on Classical Electromagnetically Induced Transparency," Phys. Rev. Lett., vol. 102, no. 5, 053901, Feb. 2009.

[19] P. Tassin, L. Zhang, T. Koschny, E. N. Economou, and C. M. Soukoulis, "Planar designs for electromagnetically induced transparency in metamaterials," Opt. Express, vol. 17, no. 7, pp. 5595-5605, March 2009.

[20] N. Liu, L. Langguth, T. Weiss, J. Kästel, M. Fleischhauer, T. Pfau, and H. Giessen, "Plasmonic analogue of electromagnetically induced transparency at the Drude damping limit," Nature Mater., vol. 8, no. 9, pp. 758-762, Sept. 2009.

[21] S.-Y. Chiam, R. Singh, C. Rockstuhl, F. Lederer, W. Zhang, and A. A. Bettiol, "Analogue of electromagnetically induced transparency in a terahertz metamaterial," Phys. Rev. B, vol. 80, no. 15, 153103, Oct. 2009.

[22] Y. Tamayama, T. Nakanishi, Y. Wakasa, T. Kanazawa, K. Sugiyama, and M. Kitano, "Electromagnetic response of a metamaterial with fieldgradient-induced transparency," Phys. Rev. B, vol. 82, no. 16, 165130, Oct. 2010.

[23] Y. Lu, J. Y. Rhee, W. H. Jang, and Y. P. Lee, "Active manipulation of plasmonic electromagnetically-induced transparency based on magnetic plasmon resonance," Opt. Express, vol. 18, no. 20, pp. 20912-20917, Aug. 2010.

[24] C. Kurter, P. Tassin, L. Zhang, T. Koschny, A. P. Zhuravel, A. V. Ustinov, S. M. Anlage, and C. M. Soukoulis, "Classical Analogue of Electromagnetically Induced Transparency with a Metal-Superconductor Hybrid Metamaterial," Phys. Rev. Lett., vol. 107, no. 4, 043901, July 2011. 
[25] Y. Tamayama, T. Nakanishi, and M. Kitano, "Variable group velocity in a metamaterial with field-gradient-induced transparency," arXiv:1109.3048 [physics:optics] 2011.

[26] C. L. G. Alzar, M. A. G. Martinez, and P. Nussenzveig, "Classical analog of electromagnetically induced transparency," Am. J. Phys., vol. 70, no. 1, pp. 37-41, Jan. 2002.

[27] I. V. Shadrivov, A. B. Kozyrev, D. W. van der Weide, and Y. S. Kivshar, "Tunable transmission and harmonic generation in nonlinear metamaterials," Appl. Phys. Lett., vol. 93, no. 16, 161903, Oct. 2008.

[28] B. Wang, J. Zhou, T. Koschny, and C. M. Soukoulis, "Nonlinear properties of split-ring resonators," Opt. Express, vol. 16, no. 20, pp. 1605816063, Sept. 2008.

[29] D. A. Powell, I. V. Shadrivov, and Y. S. Kivshar, "Nonlinear electric metamaterials," Appl. Phys. Lett., vol. 95, no. 8, 084102, Aug. 2009.

[30] D. Huang, A. Rose, E. Poutrina, S. Larouche, and D. R. Smith, "Wave mixing in nonlinear magnetic metacrystal," Appl. Phys. Lett., vol. 98, no. 20, 204102, May 2011.

[31] Z. Wang, Y. Luo, T. Jiang, Z. Wang, J. Huangfu, and L. Ran, "Harmonic Image Reconstruction Assisted by a Nonlinear Metamaterial Surface," Phys. Rev. Lett., vol. 106, no. 4, 047402, Jan. 2011.

[32] R. Yang and I. V. Shadrivov, "Double-nonlinear metamaterials," Appl. Phys. Lett., vol. 97, no. 23, 231114, Dec. 2010.

[33] P.-Y. Chen, M. Farhat, and A. Alù, "Bistable and Self-Tunable NegativeIndex Metamaterial at Optical Frequencies," Phys. Rev. Lett., vol. 106, no. 10, 105503, March 2011.

[34] M. W. Klein, C. Enkrich, M. Wegener, and S. Linden, "Second-harmonic generation from magnetic metamaterials." Science, vol. 313, no. 502, pp. 502-504, July 2006.

[35] M. W. Klein, M. Wegener, N. Feth, and S. Linden, "Experiments on second- and third-harmonic generation from magnetic metamaterials," Opt. Express, vol. 15, no. 8, pp. 5238-5247, April 2007.

[36] E. Kim, F. Wang, W. Wu, Z. Yu, and Y. Shen, "Nonlinear optical spectroscopy of photonic metamaterials," Phys. Rev. B, vol. 78, no. 11, 113102, Sept. 2008.

[37] Z. Wang, Y. Luo, L. Peng, J. Huangfu, T. Jiang, D. Wang, H. Chen, and L. Ran, "Second-harmonic generation and spectrum modulation by an active nonlinear metamaterial," Appl. Phys. Lett., vol. 94, no. 13, 134102, March 2009.

[38] A. Rose, D. Huang, and D. R. Smith, "Controlling the second-harmonic in a phase matched negative-index metamaterial," Phys. Rev. Lett., vol. 107, 063902, May 2011.

[39] M. V. Gorkunov, I. V. Shadrivov, and Y. S. Kivshar, "Enhanced parametric processes in binary metamaterials," Appl. Phys. Lett., vol. 88, no. 7, 071912, Feb. 2006.

[40] T. Kanazawa, Y. Tamayama, T. Nakanishi, and M. Kitano, "Enhancement of second harmonic generation in a doubly resonant metamaterial," Appl. Phys. Lett., vol. 99, no. 2, 024101, July 2011.

[41] E. Poutrina, D. Huang, and D. R. Smith, "Analysis of nonlinear electromagnetic metamaterials," New J. Phys., vol. 12, no. 9, 093010, Sept. 2010 . 


\section{Introduction}

Interest continues to grow in controlling electromagnetic wave propagation by using periodically or randomly arranged artificial structures made of metals, dielectrics, or other materials. When the dimensions of the constituent structures and the separation between neighboring structures are much smaller than the wavelength of electromagnetic waves, an ensemble of the structures behaves as a continuous medium for the electromagnetic waves. Such an artificial continuous medium is called a metamaterial.

The use of metallic structures as artificial media has been studied at frequencies below microwave frequencies since the late 1940s [1]. Initially, only control of the permittivity was investigated and not control of the permeability. However, Pendry et al. proposed methods for fabricating artificial magnetic media (i.e., magnetic metamaterials) in 1999 [2]. The constituent of the magnetic metamaterial is known as a split-ring resonator (SRR) and is constructed from nonmagnetic conductors. It behaves as an artificial resonant atom that has a magnetic response. Not only can the relative permeability be changed from unity, but it can also be negative as a result of the resonance. The relative permeabilities of naturally occurring media are almost unity in high frequency regions such as microwave, terahertz, and optical regions. However, this limitation on the relative permeability can be removed by using the magnetic metamaterial.

With the development of the resonant metamaterial, extraordinary media that do not occur in nature, such as media with a negative refractive indices [3], can be obtained. Concurrently, the theory of optics has been advancing rapidly. As a result, subwavelength imaging [4,5], transformation optics [6], wave propagation in epsilon-near-zero materials [7], and Brewster's no-reflection effect for chiral media $[8,9]$ have been proposed and some of them have been experimentally verified.

While the constitutive parameters (e.g., permittivity and permeability) for a monochromatic wave can be controlled by using metamaterials with a unit cell consisting of a single resonant element, other characteristics can also be controlled by using metamaterials composed of coupled resonators. In this paper, we describe group velocity control and efficient second harmonic generation (SHG) in coupled-resonator-based metamaterials. The first part of the paper considers metamaterials that mimic electromagnetically induced transparency (EIT) $[10,11,12]$. After describing EIT and its electrical circuit model, which consists of a coupled resonant circuit, we present a few EIT-like metamaterials. The second part of the paper deals with SHG in resonant metamaterials with nonlinear elements. We first describe a method for enhancing nonlinearity of nonlinear elements in resonant metamaterials and then present an SHG experiment in a singly resonant metamaterial. Finally, we present a method for further enhancing SHG in a doubly resonant metamaterial that consists of coupled resonant structures. 


\section{EIT-like metamaterials}

The phenomenon of EIT has attracted considerable attention in recent years as a means for controlling the group velocity of electromagnetic waves [10, $11,12]$. It is a quantum interference phenomenon that occurs in $\Lambda$-type three-state atoms that interact with two electromagnetic waves: a probe wave, which is tuned to the transition between the ground state and the common excited state of the atom, and a coupling wave, which is tuned to the transition between another lower state and the excited state. The atoms absorb the probe wave in the absence of the coupling wave, but this absorption is suppressed in a narrow frequency range when the coupling wave is present. The Kramers-Kronig relations [13] can be used to show that a steep dispersion can be generated for the probe wave in this frequency range. This steep dispersion reduces the group velocity of the probe wave because the group velocity is given by $v_{\mathrm{g}}=\mathrm{d} \omega / \mathrm{d} k=c_{0} /[n+\omega(\mathrm{d} n / \mathrm{d} \omega)]$, where $\omega$ is the angular frequency, $k$ is the wavenumber, $c_{0}$ is the speed of light in vacuum, and $n(\omega)$ is the refractive index. In fact, a group velocity of $17 \mathrm{~m} / \mathrm{s} \sim 10^{-7} c_{0}$ has been observed using atomic transitions [11]. In addition, the bandwidth of the transmission window, or the steepness of the dispersion, depends on the Rabi frequency of the coupling wave, and thus the group velocity can be controlled by varying the intensity of the coupling wave.

Since rather complex experimental setups are required to achieve EIT, several studies have sought to mimic the effect in classical systems, especially in metamaterials $[14,15,16,17,18,19,20,21,22,23,24,25]$. To understand the theory of EIT-like metamaterials, it is useful to briefly review a classical model for EIT. Figure 1 (a) shows an electrical circuit model of EIT [26]. The circuit consists of two inductor-capacitor-resistor resonant circuits, one of which has a low $Q$ value and the other has a high $Q$ value (i.e., $R_{1} \gg R_{2}$ ). These resonant circuits are coupled via a mutual inductance $M$. The charge

(a)

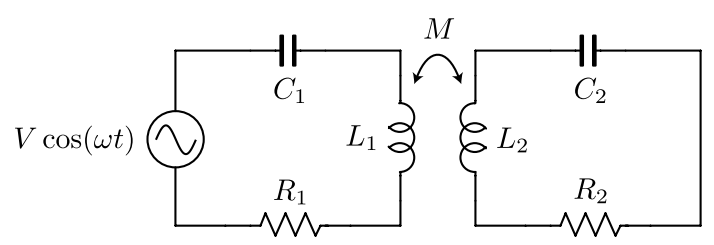

(b)

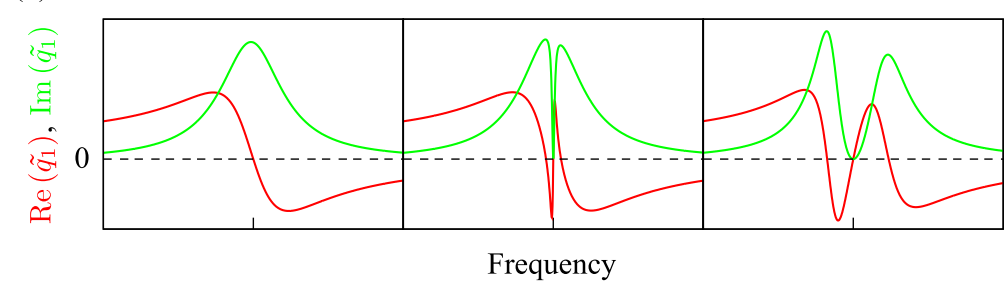

Fig. 1. (a) Electrical circuit model of EIT. (b) Frequency dependence of the complex amplitude $\tilde{q_{1}}$ of the charge stored in capacitor $C_{1}$ for the cases when the mutual inductance $M$ is zero (left panel), small (center panel), and large (right panel). 
$q_{1}$ stored in the capacitor in the low- $Q$ circuit is written as

$$
q_{1}=-\frac{V}{2 L_{1}} \frac{\omega^{2}+\mathrm{i} \gamma_{2} \omega-\omega_{2}^{2}}{\left(\omega^{2}+\mathrm{i} \gamma_{1}-\omega_{1}^{2}\right)\left(\omega^{2}+\mathrm{i} \gamma_{2} \omega-\omega_{2}^{2}\right)-K^{2} \omega^{4}} \mathrm{e}^{-\mathrm{i} \omega t}+\text { c.c., }
$$

where $\omega_{i}=1 / \sqrt{L_{i} C_{i}}, \gamma_{i}=R_{i} / L_{i}(i=1,2), K=M / \sqrt{L_{1} L_{2}}$, and c.c. indicates the complex conjugate of the preceding term. Figure 1 (b) shows the complex amplitude of $q_{1}$ as a function of frequency. A simple resonant characteristic is observed for $M=0$, whereas a dip appears in the resonant spectrum for $M \neq 0$. The bandwidth of the dip becomes wider as $M$ increases. This behavior is similar to that of the electric susceptibility for EIT. Due to destructive interference between the current in the low- $Q$ circuit induced by the voltage source and that induced by the high- $Q$ circuit, the current in the low- $Q$ circuit may vanish. Therefore, if a unit cell of a metamaterial consists of a low- $Q$ and a high- $Q$ resonator coupled with each other and an incident electromagnetic wave can directly interact only with the low- $Q$ resonator, the metamaterial behaves as an EIT-like medium.

The EIT-like metamaterial developed for the first time is an array of asymmetrically split rings (see Fig. 2 (a) [14]). An experiment was performed in the microwave region. The rings were fabricated on a printed circuit board. There are two kinds of resonant modes in the asymmetrically split rings. The current flows in the two parts of the ring are parallel for one mode (low- $Q$

(a)

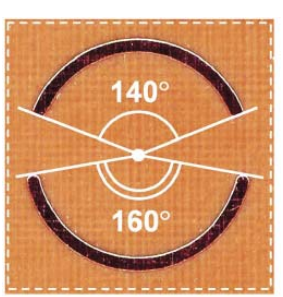

(b)

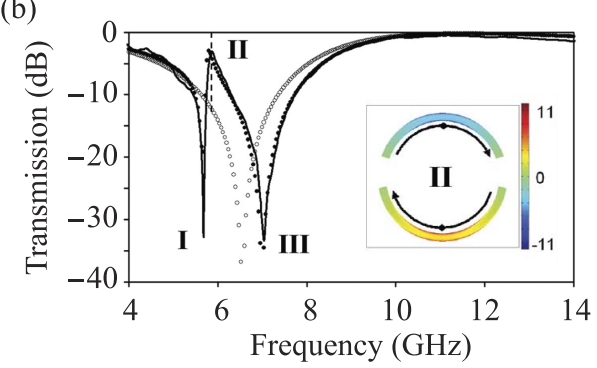

(c)

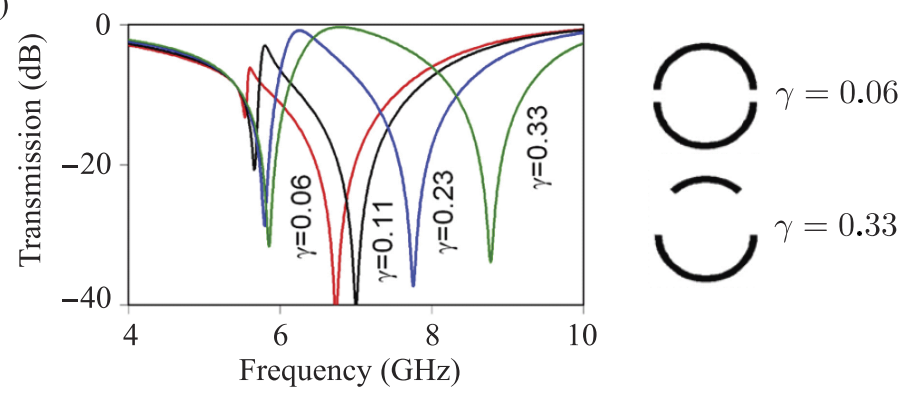

Fig. 2. (a) Unit structure of an asymmetrically split ring. (b) Transmission spectrum of the metamaterial: solid line - experiment, filled circles - theory, open circles - theory for reference structure with symmetrically split rings. The inset shows the current flow at the transmission peak frequency. (c) Dependence of the transmission spectrum on the asymmetry parameter $\gamma$. Geometries of rings with $\gamma=0.06$ and 0.33 are also shown. Adapted from Ref. [14]. 
mode) and antiparallel for the other mode (high- $Q$ mode). The former mode can be excited by a horizontally polarized electric field. Although the latter mode is inaccessible in symmetrically split rings, the coupling between these two modes is induced due to symmetry breaking in the shape of the rings and the latter mode can be excited through the coupling in asymmetrically split rings. The response of the asymmetrically split ring to the horizontally polarized wave is similar to the classical model of EIT shown in Fig. 1(a); thus, an array of asymmetrically split rings behaves as an EITlike medium. Figure 2 (b) shows transmission spectra of metamaterials that consist of asymmetrically split rings and symmetrically split rings. An absorption line is observed for the symmetrically split rings, whereas a sharp EIT-like transmission peak appears near $6 \mathrm{GHz}$ in the absorption line for the asymmetrically split rings. Figure 2 (c) shows the dependence of the transmission spectrum on the asymmetry parameter $\gamma$. A larger $\gamma$ implies that the structure is more asymmetric; i.e., that there is stronger coupling between the two modes. As $\gamma$ increases, the transmission bandwidth increases, while the transmission peak frequency decreases.

A metamaterial that mimics EIT has also been proposed [15] and realized [20] in the optical region. This EIT-like optical metamaterial is shown in Fig. 3 (a). It was fabricated by using nanofabrication stacking techniques. The unit cell of the optical metamaterial is composed of two functional layers: a gold bar stacked above two symmetric gold bars. The top gold bar (in red) serves as an electric dipole oscillator (low- $Q$ resonator) and the bottom gold bar pair (in green) serves as an electric quadrupole oscillator (high$Q$ resonator). A $y$-polarized incident electric field can directly excite only

(a)

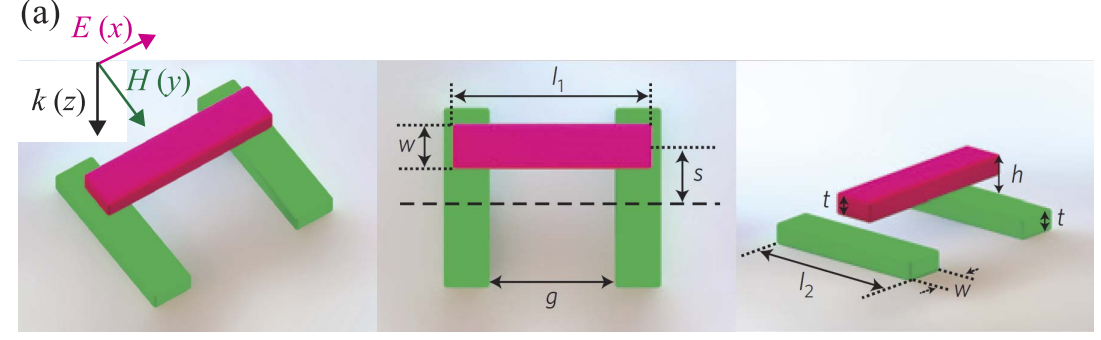

(b)

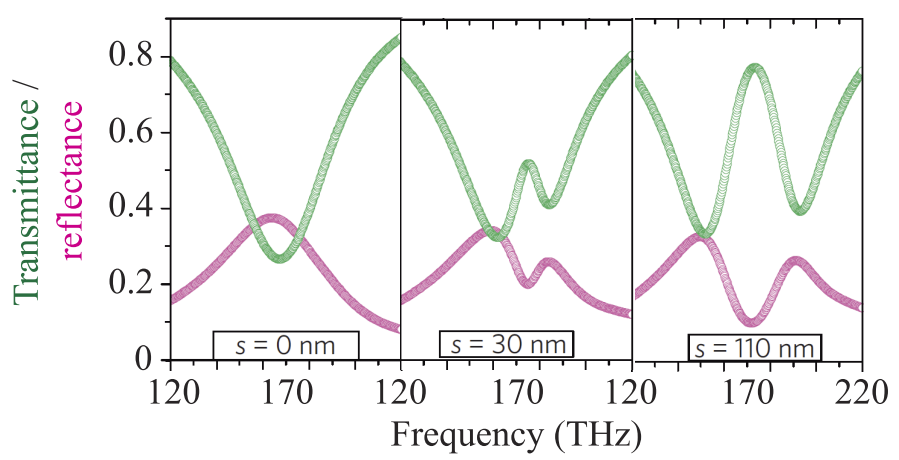

Fig. 3. (a) Unit structure of an optical EIT-like metamaterial. (b) Transmission (green) and reflection (red) spectra of the metamaterial for three different lateral shifts $s$. Adapted from Ref. [20]. 
the electric dipole oscillator; the coupling between these two oscillators is induced when a structural asymmetry is introduced. The asymmetry is controlled by varying the lateral displacement $s$ of the top bar with respect to the symmetry axis of the bottom bars. Figure 3 (b) shows transmission and reflection spectra of the metamaterial. An EIT-like transmission window is observed in the absorption spectrum for $s \neq 0$. With increasing $s$, the transmission bandwidth increases and the transmission peak frequency remains unchanged.

In order to vary the transmission bandwidth, or the group velocity at the transparency frequency, in the above-mentioned metamaterials, the geometrical parameters of the metamaterials need to be altered. Thus, it is not practical to variably control the group velocity in these metamaterials. To overcome this problem, the metamaterial shown in Fig. 4 (a) was proposed [22, 25]. This metamaterial has two resonant modes: an electric dipole resonance (its current flow is represented by the solid arrows) and a magnetic quadrupole-like resonance (represented by the dashed arrows). The latter mode has a higher $Q$-value than the former mode. An $x$-polarized incident electric field can interact only with the electric dipole oscillator. The

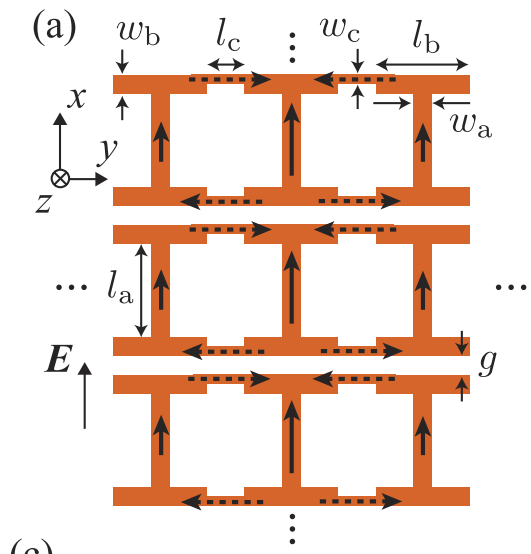

(c)

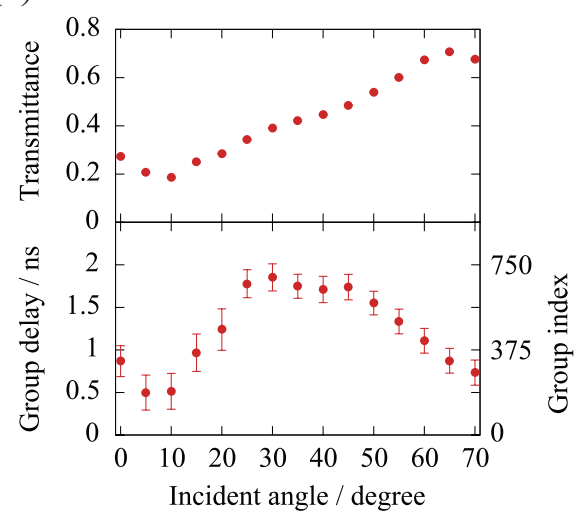

(b)

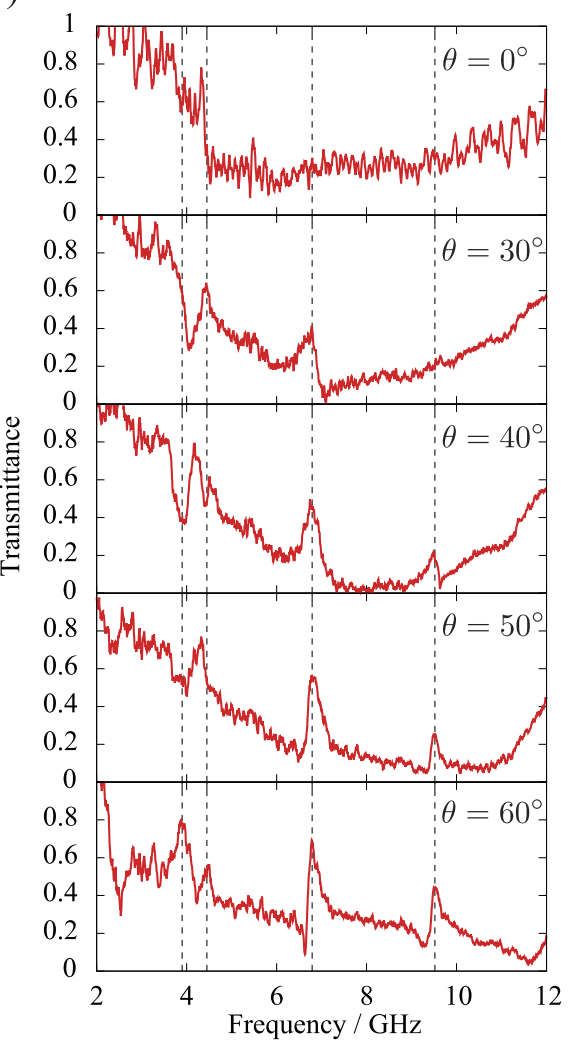

Fig. 4. (a) Schematic of metamaterial with field-gradientinduced transparency. (b) Transmission spectra for various incident angles $\theta$. The vertical dashed lines serve as guides to the eye for comparing the transmission peak frequencies for different $\theta$. (c) Transmittance and group delay as a function of $\theta$. 
coupling between these two resonant modes is induced when the incident electromagnetic field has a field gradient in the $y$ direction. While structural asymmetry induces the coupling between the low- $Q$ and high- $Q$ modes in the above-mentioned EIT-like metamaterials, asymmetry of the electromagnetic field induces the coupling in this metamaterial. Since the transparency phenomenon is induced by the field gradient, the EIT-like phenomenon is referred to as field-gradient-induced transparency. To achieve variable control of the group velocity in this metamaterial, the coupling strength between the two resonant modes needs to be controlled. The coupling strength depends on the gradient of the $x$ component of the incident electric field in the $y$ direction. Thus, the group velocity can be varied by changing the incident angle of the plane wave.

Figure 4 (b) shows transmission spectra of the metamaterial obtained at five different incident angles. Only a broad absorption line is observed for normal incidence, whereas three EIT-like narrow transmission windows appear at about $4.2,6.79$, and $9.52 \mathrm{GHz}$ in the absorption line for oblique incidence. The transmission peak at $6.79 \mathrm{GHz}$ corresponds to the magnetic quadrupole-like resonance. Its peak frequency varies little as the incident angle is changed; therefore, the transmission window at $6.79 \mathrm{GHz}$ is suitable for controlling the group velocity. Figure 4 (c) shows the transmittance and group delay of the metamaterial for a microwave pulse with a carrier frequency of $6.79 \mathrm{GHz}$. The delay of the pulse center was regarded as being the group delay in this experiment. With increasing the incident angle, the transmittance increases monotonically, while the group delay initially increases, reaches a maximum at about $30^{\circ}$, and then decreases. This observation is consistent with the characteristics of EIT. The group delay is tunable in the range 0.50 to $1.85 \mathrm{~ns}$, which corresponds to a group index from 188 to 694 . In addition, the transmitted pulse does not exhibit any significant distortion (see Ref. [25]).

\section{Nonlinear metamaterials for SHG}

Metamaterials have been employed to control linear dispersion of media. An EIT-like metamaterial is a typical example of a highly dispersive media. It is also possible to realize nonlinear media by introducing nonlinear elements into constituents of metamaterials. Pendry et al. [2] theoretically predicted that high nonlinearities could be realized in resonant metamaterials such as SRRs by placing nonlinear elements at locations where electric (or magnetic) field is concentrated due to the resonance effect. Nonlinear metamaterials have been applied to applications such as property-tunable metamaterials [27, 28, 29], frequency mixing [30], imaging beyond the diffraction limit [31], and bistable media $[28,32,33]$. In this section, we review some papers on SHG in nonlinear metamaterials with a single resonant structures in a unit cell $[27,34,35,36,37,38]$. We also describe more efficient methods for generating second harmonic ( $\mathrm{SH}$ ) waves by introducing a coupled-resonatorbased metamaterial, which simultaneously resonates at the fundamental and 
SH frequencies $[39,40]$.

\subsection{SHG in singly resonant metamaterials}

Enhancement of SHG in a nonlinear metamaterial was first demonstrated in the optical region [34]. Figure 5 (a) shows structures of SRRs and transmission spectra for normally incident waves with different polarizations. The transmission spectrum for horizontally polarized waves (upper curve) shows a resonant dip at a wavelength of $1.5 \mu \mathrm{m}$. The resonant mode is formed by circulating current along the SRR and the current generates strong magnetic fields perpendicular to the SRR. In the presence of oscillating magnetic fields $\boldsymbol{B}$ (and oscillating electric fields $\boldsymbol{E}$ ), electrons moving at a velocity of $\boldsymbol{v}$ are driven by the Lorentz force $\boldsymbol{F}=q(\boldsymbol{E}+\boldsymbol{v} \times \boldsymbol{B})$. The latter (magnetic) term in $\boldsymbol{F}$, which is usually negligibly small, plays an important role in generating $\mathrm{SH}$ waves in strong magnetic fields. Electrons moving in the direction of $\boldsymbol{E}$ are subjected to the Lorentz force and oscillate at a frequency of $2 \omega$ in the direction of $\boldsymbol{E} \times \boldsymbol{B}$. For free propagating waves, SH oscillations occur in the longitudinal direction and thus cannot radiate $\mathrm{SH}$ waves in the forward direction. On the other hand, in the presence of the SRRs, SH oscillations occur in the transverse direction (i.e., vertical direction in Fig. $5(\mathrm{a})$ ) due to the strong magnetic fields induced by the resonant currents in the SRRs. Consequently, substantial SH waves are generated in the forward direction. For vertically polarized incident waves, only $1.3 \%$ of the $\mathrm{SH}$ signal with respect to that for horizontally polarized waves was observed due to the absence of the resonant magnetic field.

In the microwave region, varactor (variable capacitance) diodes are often used as nonlinear elements. Unlike normal capacitors, the capacitance $C(q)$ of the varactor diodes depends on the stored charge $q$. The diode voltage can be represented as $v_{\mathrm{C}}=q / C(q) \simeq q / C+\alpha q^{2}$, where the second term contributes to SHG. If varactor diodes are introduced into resonant metamaterials at locations where electric fields are concentrated, efficient SHG can be expected. In the case of SRRs, varactor diodes should be located across the gap in the split ring, as shown in Fig. 5 (b) [27]. With the help of the circuit model shown in Fig. 6 (a), which is widely utilized for singly
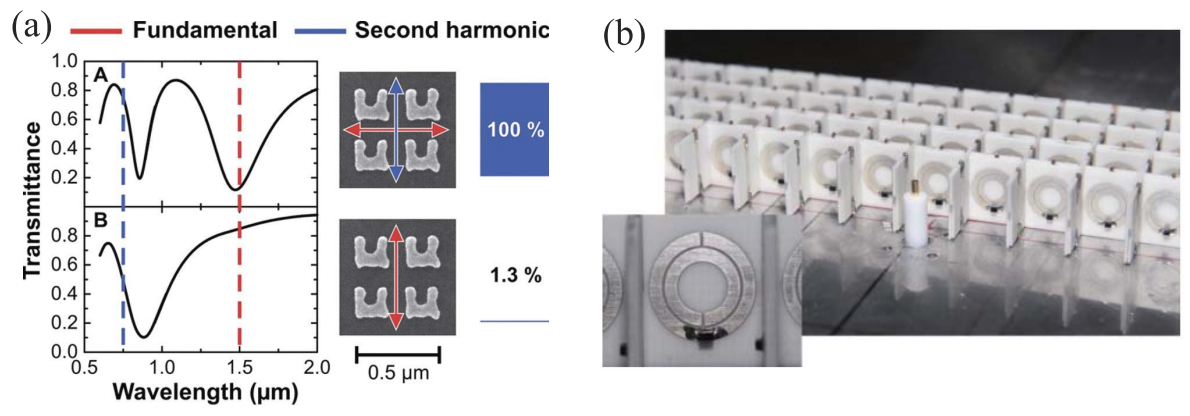

Fig. 5. Enhancement of SHG process (a) in optical region (adapted from Ref. [34]) and (b) in microwave region (adapted from Ref. [27]). 

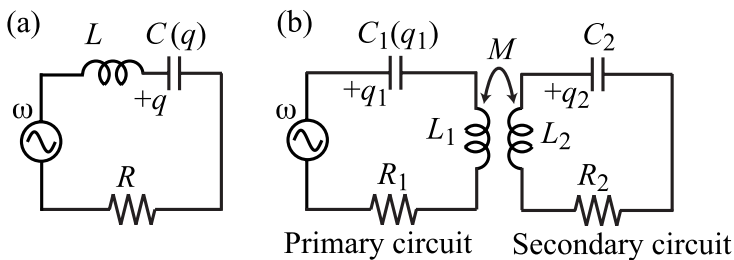

Fig. 6. Circuit models of (a) singly resonant and (b) doubly resonant metamaterials.

resonant metamaterials, the circulating current at $2 \omega$ can be easily derived as

$$
|I(2 \omega)|=\frac{|\alpha| V^{2}}{\omega^{2}\left|Z(2 \omega) Z(\omega)^{2}\right|},
$$

where $Z(\omega)$ is the impedance of the circuit and $V$ is the amplitude of electromotive voltage induced by the magnetic field [41]. When $\omega$ is tuned to the resonant angular frequency $\omega_{0}=1 / \sqrt{L C},|Z(\omega)|$ has a minimum value and $|I(2 \omega)|$ is maximized. Strong SH waves are radiated from magnetic moments induced by the $\mathrm{SH}$ current in a singly resonant metamaterial.

\subsection{SHG in doubly resonant metamaterials}

Equation (2) shows that the impedance for $2 \omega,|Z(2 \omega)|$, also affects the amplitude of the SH current. In a singly resonant metamaterial that resonates only at the frequency of the fundamental wave, $|Z(2 \omega)|$ is relatively large because the SH frequency is off-resonance. Hence, the SH current $|I(2 \omega)|$ could be further enhanced by reducing $|Z(2 \omega)|$ in some way. That is, more efficient SHG could be achieved when the resonant conditions for both the fundamental and SH frequencies are satisfied. A metamaterial with two resonant modes (i.e., a doubly resonant metamaterial) has the potential to simultaneously realize both resonant conditions [39].

The double cut-wire structure shown in Fig. 7 (a) is an example for satisfying the doubly resonant condition. The metamaterial is composed of two resonant structures: a primary resonator [Fig. 7 (b)] and a secondary resonator [Fig. 7 (c)] [40]. A varactor diode is loaded at the center of the cutwire structure of the primary resonator. When a periodic boundary condition is imposed in the $x$ direction, both the primary and secondary resonators can be modeled as series inductor-capacitor resonant circuits. When the two structures are close to each other, as shown in Fig. 7 (a), they will be magnetically coupled and they can be modeled as a magnetically coupled resonant circuit, as shown in Fig. 6 (b). Note that the capacitance of the primary resonator is nonlinear. The resonant angular frequencies of the two resonators, $\omega_{1}=1 / \sqrt{L_{1} C_{1}}$, and $\omega_{2}=1 / \sqrt{L_{2} C_{2}}$, are assumed to satisfy $2 \omega_{1} \simeq \omega_{2}$. When the primary circuit is excited at $\omega=\omega_{1}$, the SH electromotive voltage is generated in the nonlinear capacitor $C_{1}$ and excites resonance in the secondary circuit through the magnetic coupling $M$. Due to the double resonance, the SHG efficiency will be higher than without the secondary resonator.

SHG enhancement can be confirmed by the following circuit analysis. The 


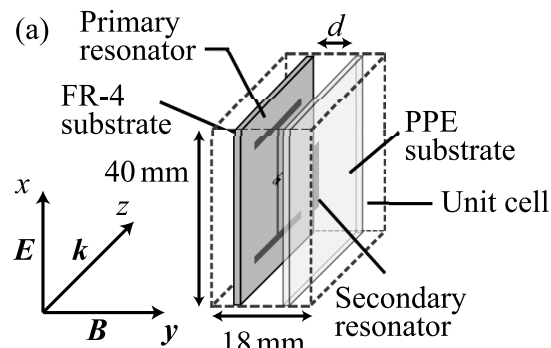

(b)

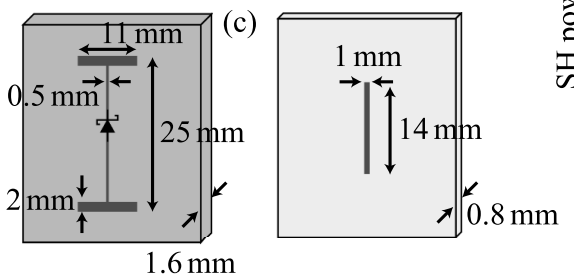

(d)

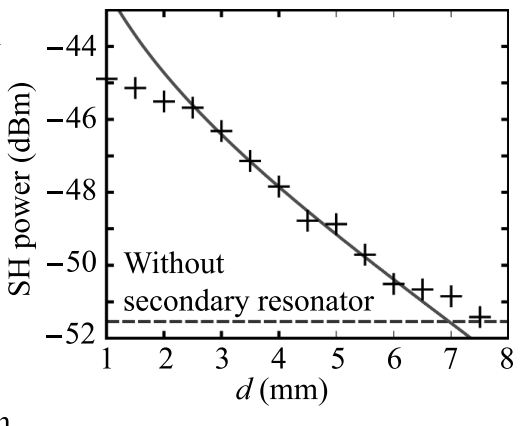

Fig. 7. (a) Unit cell of a doubly resonant metamaterial and structures of (b) primary and (c) secondary resonators. (d) SH power versus $d$ at the fundamental frequency of $3.4 \mathrm{GHz}$.

$\mathrm{SH}$ current in the secondary resonator is calculated as

$$
\left|I_{2}(2 \omega)\right| \approx \frac{|\alpha M| V^{2}}{\omega\left|Z_{1}(2 \omega) Z_{2}(2 \omega) Z_{1}(\omega)^{2}\right|},
$$

where $Z_{1}(\omega)$ and $Z_{2}(\omega)$ are the impedances of the primary and secondary circuits, respectively. If the ratio between $I(2 \omega)$ in Eq. (2) and $I_{2}(2 \omega)$ in Eq. (3) is defined as $\beta \equiv\left|I_{2}(2 \omega) / I(2 \omega)\right| \approx\left|\omega M / Z_{2}(2 \omega)\right|$, then $\beta^{2}$ can be regarded as the SHG enhancement factor due to the presence of the secondary resonator. Because of the resonance condition $2 \omega_{1} \simeq \omega_{2}, \beta^{2}$ will be large.

Figure $7(\mathrm{~d})$ shows experimental results for the SH power for various separations $d$ between the two resonators. For comparison, the dashed line indicates the SH power obtained without the secondary resonator. The $\mathrm{SH}$ power increases with decreasing $d$ (i.e., increasing the coupling $M$ ). This is consistent with Eq. (3), derived from the circuit analysis. For $d=1 \mathrm{~mm}$, the SHG efficiency with the doubly resonant metamaterial is $7 \mathrm{~dB}$ higher than that without the secondary resonator.

\section{Conclusion}

We have discussed electromagnetic wave propagation in metamaterials composed of two coupled resonators. More parameters can be controlled in coupled-resonator-based metamaterials than in metamaterials with a unit cell of a single resonant element. As examples of coupled-resonator-based metamaterials, we described EIT-like metamaterials and a doubly resonant nonlinear metamaterial, which can be respectively used for controlling group velocity and enhancing SHG. The first part of this paper presented a method for realizing metamaterials that mimic EIT and described some EIT-like metamaterials. We also showed an experiment that realized variable control of the group velocity in a metamaterial with field-gradient-induced transparency. 
The second part of the paper considered SHG in nonlinear metamaterials. We presented experiments in which SHG was enhanced in singly resonant metamaterials at optical and microwave frequencies and then showed that the SHG efficiency can be further enhanced in a doubly resonant metamaterial due to resonance at the fundamental and $\mathrm{SH}$ frequencies.

Electrical circuit models of the EIT-like metamaterial and doubly resonant metamaterial have the same configurations of the circuit elements, as shown in Figs. 1 (a) and 6 (b), although the conditions for the circuit constants are different. This implies that other novel phenomena may be discovered by considering various kinds of conditions for the circuit constants of the two coupled resonant circuits. It is also important to investigate the electromagnetic responses of metamaterials that consist of more than two coupled resonators.

\section{Acknowledgments}

This research was supported in part by Grants-in-Aid for Scientific Research (Grant Nos. 22109004 and 22560041), by the Global COE Program "Photonics and Electronics Science and Engineering" at Kyoto University, and by a research grant from the Murata Science Foundation. One of the authors (Y.T.) would like to acknowledge the support of a Research Fellowship of the Japan Society for the Promotion of Science for Young Scientists.

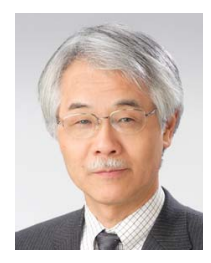

\section{Masao Kitano}

was born in Kyoto, Japan, on August 5, 1952. He received the B.S., M.S., and $\mathrm{PhD}$ degrees in electronic engineering from Kyoto University, Kyoto, Japan, in 1975, 1977, and 1984, respectively. In 1977 he joined the Department of Electronics, Kyoto University and since 1999, he is a Professor at the Department of Electronic Science and Engineering, Kyoto University. He spent the academic years 1984-1986 on leave at the Department of Physics, Princeton University. His research interest includes quantum optics, nearfield optics, optical pumping, electromagnetism. He received the Matsuo Science Prize in 2007. Dr. Kitano is a member of the Institute of the Electronics, Information and Communication Engineers, American Physical Society, the Physical Society of Japan, the Laser Society of Japan, and the Japan Society of Applied Physics.

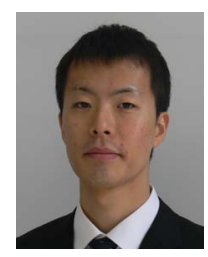

\section{Yasuhiro Tamayama}

was born in Osaka, Japan, on October 27, 1981. He received the B.S. degree in electrical and electronic engineering, and the M.S. and Ph.D. degrees in electronic science and engineering from Kyoto University, Kyoto, Japan, in 2004, 2006, and 2011, respectively. From 2006 to 2008, he worked for Konica Minolta Sensing, Inc. He has been a Research Fellow of the Japan Society for the Promotion of Science since 2010 and a postdoctoral fellow at Kyoto University since 2011. His current research interest is in electromagnetic metamaterials and electromagnetic wave propagation. Dr. Tamayama is a member of the Physical Society of Japan and the Institute of Electronics, Information and Communication Engineers. 


\section{Toshihiro Nakanishi}

was born in Mie Prefecture, Japan, on June 22, 1976. He received the B.S. and M.S. degrees in electronic science and engineering from Kyoto University, Kyoto, Japan, in 2000 and 2002 respectively. In 2003, he joined the Department of Electronic Science and Engineering at Kyoto University. His work has been on quantum electronics: quantum measurement; quantum optics; extraordinary light propagation. He is currently engaging in the development of metamaterials, especially in microwave region. Mr. Nakanishi is a member of the Physical Society of Japan and a member of the Institute of Electronics, Information and Communication Engineers. 